\title{
1 Genomic insights into the phylogeny and biomass-degrading enzymes of rumen ciliates
}

2 Zongjun $\mathrm{Li}^{1} \uparrow$, Xiangnan Wang ${ }^{1} \uparrow$, Yu Zhang ${ }^{1} \uparrow$, Zhongtang $\mathrm{Yu}^{2} \uparrow$, Tingting Zhang ${ }^{1} \uparrow$, Xuelei

$3 \mathrm{Dai}^{1}$, Xiangyu $\mathrm{Pan}^{1}$, Ruoxi Jing ${ }^{1,3}$, Yueyang Yan $^{1}$, Yangfan $\mathrm{Liu}^{1,3}$, Shan $\mathrm{Gao}^{1}$, $\mathrm{Fei}^{1} i^{4}$, Youqin

4 Huang ${ }^{1,4}$, Jian Tian $^{5}$, Junhu Yao ${ }^{1}$, XvPeng Xing ${ }^{6}$, Tao Shi ${ }^{1}$, Jifeng Ning ${ }^{7}$, Bin Yao ${ }^{5}$, Huoqing

$5 \quad$ Huang $^{5 *}$, Yu Jiang ${ }^{1 *}$

6

$7 \quad{ }^{1}$ Center for Ruminant Genetics and Evolution, College of Animal Science and Technology,

8 Northwest A\&F University, Yangling 712100, China

$9 \quad{ }^{2}$ Department of Animal Sciences, The Ohio State University, Columbus, OH 43210, USA

$10{ }^{3}$ Key Laboratory of Agricultural Animal Genetics, Breeding, and Reproduction of Ministry of

11 Education, College of Animal Science and Technology, Huazhong Agricultural University,

12 Wuhan, 430070, China

$13{ }^{4}$ State Key Laboratory of Grassland Agro-ecosystems, College of Pastoral Agriculture

14 Science and Technology, Lanzhou University, Lanzhou, 730020, China

$15{ }^{5}$ State Key Laboratory of Animal Nutrition, Institute of Animal Sciences, Chinese Academy

16 of Agricultural Sciences, Beijing 100193, China

$17 \quad{ }^{6}$ Key Laboratory of Animal Biotechnology of the Ministry of Agriculture, College of

18 Veterinary Medicine, Northwest A\&F University, Yangling, 712100, China

$19{ }^{7}$ College of Information Engineering, Northwest A\&F University, Yangling, 712100, China

$21 \dagger$ These authors contributed equally.

$22{ }^{*}$ Corresponding author. E-mail: yu.jiang@nwafu.edu.cn, huanghuoqing@caas.cn 


\section{Abstract}

25 Understanding the biodiversity and genetics of the gut microbiome has important

26 implications for host physiology. One underexplored and elusive group is ciliated protozoa,

27 which play crucial roles in regulating gut microbial interactions. Integrating single-cell

28 sequencing and an assembly-and-identification pipeline, we acquired 52 high-quality ciliate

29 genomes of 22 rumen morphospecies for all major abundant clades. With these genomes, we

30 firstly resolved the taxonomic and phylogenetic framework that reclassified them into 19

31 species spanning 13 genera and reassigned the genus Dasytricha from Isotrichidae to a new

32 family Dasytrichidae. Via extensive horizontal gene transfer and gene family expansion,

33 rumen ciliates possess a broad array of enzymes to synergistically degrade plant and

34 microbial carbohydrates. In particular, $\sim 80 \%$ of the degrading enzymes in Diplodiniinae and

35 Ophryoscolecinae act on plant cell wall, and the high activities of their cellulase,

36 xylanase and lysozyme reflect the potential of ciliate enzymes for biomass-

37 conversion. Additionally, the new ciliate dataset greatly facilitated the rumen metagenomic

38 analyses by allowing $\sim 12 \%$ of reads to be classified. 


\section{Introduction}

41 The genomes of hundreds of thousands of gut prokaryotes have been sequenced, which have

42 greatly advanced our understanding of their taxonomic and functional diversity and important

43 roles in host health and nutrition ${ }^{1-3}$. Trichostome ciliates (of the phylum Ciliophora, class

44 Litostomatea) are ubiquitous in the gut microbiome of vertebrates ${ }^{4,5}$, and they serve crucial roles in modulating the gut microbiome through predation, competition, and symbiosis ${ }^{6}$.

46 However, genomic research on gut ciliates has lagged ${ }^{1}$, and by far only Entodinium caudatum, one of the most common species in the rumen of ruminants, has been subjected to genomic study ${ }^{7}$. The lack of genomic information on the ciliate has become an obstacle for holistic studies of the gut microbiome, rumen microbiome in particular, as ciliates can account for up to $50 \%$ of the total rumen microbial biomass and approximately 200 morphospecies have been described in the rumen ${ }^{6}$.

Despite their prominence in the rumen ecosystem and extensive previous research over one century $^{8}$, much remains to be learned about rumen ciliates ${ }^{9,10}$. Based on morphological features, rumen ciliates are primarily classified into two clades: family Isotrichidae in the order Vestibuliferida (with cilia covering the entire cell surface) and family Ophryoscolecidae in the order Entodiniomorphida (with localized zones of cilia including one or two adoral cilia zone $)^{4,6}$. It has been recognized, however, taxonomic identification and classification of ciliates based on morphological features are challenging ${ }^{6,11}$, as the same species can exhibit a few different morphotypes ${ }^{12}$. The $18 \mathrm{~S}$ rRNA gene of ciliates has been used to aid species identification $^{13}$, but it has several limitations in proposing a new taxonomic framework, including topological discrepancies even at the subfamily level ${ }^{5,14}$ and too conserved to uncover cryptic species ${ }^{15}$.

It has also been challenging to determine or investigate the actual metabolism of rumen ciliates because of the lack of and inability to establish axenic cultures. As a result, our 
knowledge of rumen ciliate metabolism can only be inferred from studies using monocultures (that contain bacteria and other microbes) or defaunation (complete removal of rumen protozoa chemically or through physical isolation), both of which introduce confounding effects of other microbes and residual effects ${ }^{10,16}$. Recent genomic and transcriptomic studies provided new insights into the metabolism and physiology of several species of rumen ciliates $^{7,17}$. However, the functional diversification and evolutionary mechanisms underlying most rumen ciliates linkages remain undetermined. Additionally, the unique genome structures (e.g., nuclear dimorphism, chromosomal fragmentation) make ciliates valuable model organisms to understand many important fundamental molecular and cellular processes and features, such as catalytic RNA, telomerase, and histone acetylation ${ }^{18,19}$. However, most knowledge comes from a few free-living aerobic ciliate species within the classes Oligohymenophorea and Spirotrichea ${ }^{20,21}$, while rumen ciliates, which are anaerobic and symbiotic, are exclusively found in the class Litostomatea.

In this study we de novo assembled and analyzed 52 high-quality single-ciliate-cell amplified genomes (SAGs) from 22 rumen morphospecies, which were reclassified into 19 species spanning 13 genera and three families in the newly genome-based taxonomy. One assembly-and-identification pipeline was developed to help improve the quality and accuracy of genome assembly of ciliates. This unparalleled genome catalog of rumen ciliates greatly facilitated and/or enabled better understanding and determination of their taxonomy, phylogeny, metabolism, and ecology.

\section{Results}

\section{Recovering single-ciliate-cell amplified genomes}

We sequenced the DNA amplified from 69 individual single ciliate cells isolated from fresh rumen fluid sampled from 15 cattle and goats (Supplementary Table 1). They represented 22 ciliate morphospecies (Fig. 1 and Supplementary Fig. 1-3) belonging to 11 prevalent and 
90

abundant morphogenera, which represent $\sim 97 \%$ of the genus-level abundance across our collected samples and published studies (Supplementary Table 2). We also sequenced the amplified transcriptome ${ }^{22}$ of 24 of the isolated single-ciliate-cells and 14 rumen metatranscriptomes for de novo gene prediction (Supplementary Fig. 4).

Because rumen ciliate cells carry other microbes as endosymbionts or engulfed microbes (primarily bacteria) and because the DNA of the latter can also be amplified and sequenced, we developed an assembly-and-identification pipeline to recover and identify ciliate macronuclear sequences (Fig. 1 and Supplementary Fig. 4). Briefly, using contigs assembled from the $2.5 \mathrm{~Tb}$ sequences from the single cells and the $\sim 17 \mathrm{~Tb}$ publicly available rumen metagenome (Supplementary Table 3), we constructed an $18 \mathrm{~Gb}$ assembled dataset of contigs of rumen ciliate macronuclear, all of which were capped on both or one end with telomeric repeats ((CCCCAAT)n). This dataset was searched for the contigs that did not have any telomeres in the single-ciliate assembled contigs (see Methods). Additionally, we performed hybrid assembly and deep sequencing to improve the assembly quality (Supplementary Fig. 5). Of the 69 SAGs we assembled (Supplementary Table 4), 52 had $\geq 80 \%$ of the 171 Alveolata conserved marker genes ${ }^{23}$ and were considered "high-quality", while 17 had $<80 \%$ of the Alveolata conserved marker genes. The 52 high-quality SAGs were used for further analysis. Among the 52 SAGs, the telomere-capped contigs ranged from 14\% to $92 \%$ (mean 61\%) of the total contigs (Supplementary Table 4). One of the SAGs of Oph. caudatus (referred to as SAGT3) had the highest number (26,497 to be exact) of complete chromosomes (contigs capped with telomere at both ends), plus another 33,578 contigs that were capped with telomere at only one end. The chromosome number of $O p h$. caudatus is at least 43,286 , which is much more than the known large number of chromosomes in ciliates, Oxytricha trifallax $(16,000)$ and Halteria grandinella $(23,000)^{20,24}$.

\section{Deriving a robust genome-based taxonomy and phylogeny}


115 A robust taxonomy is an organizing principle of biology to communicate scientific results

116 and describe biodiversity ${ }^{25,26}$. Using the 52 SAGs, we established a robust genome-based

117 taxonomic framework of rumen ciliates that overcomes the uncertainty inherent of the

118 morphology-based taxonomy.

119 Whole-genome average nucleotide identity (ANI) has emerged as a widely accepted 120 method for circumscribing prokaryotic species, with 95\% ANI as a species boundary ${ }^{26,27}$. We

121 found a clear ANI discontinuity among the SAGs: $>97 \%$ or $<92 \%$ (Fig. 2a-b) and considered ANI $>97 \%$ and $<92 \%$ for intra-species and inter-species cutoffs, respectively. Based on the above ANI cutoff values, we found four pairs of synonymic species in Ophryoscolecidae

124 (Fig. 2a and Supplementary Fig. 6). This is exemplified by Oph. caudatus, Oph. purkynjei and Oph. bicinctus (Fig. 1v-x and Supplementary Fig. 3) being classified as a single species, although Oph. caudatus was isolated from sheep and goats with a long main caudal spine and three circles of secondary caudal spines, whereas Oph. purkynjei and Oph. bicinctus were isolated from cattle with a short main caudal spine and two circles of secondary caudal spines, respectively ${ }^{6}$. Similarly, Dip. anisacanthum and Dip. dentaum, Dip. flabellum aspinatum and Dip. flabellum monospinatum, Ost.gracile and Ost. venustum were synonymy, respectively (Fig. 2a and Supplementary Fig. 1). These suggest that the shape of the main caudal spine, the number of secondary caudal spines, and habitat host are not reliably taxonomic worth for Ophryoscolecidae. On the other hand, despite having morphological

134 similarity, two new cryptic species of Isotrichidae were identified (ANI $<92 \%$, Fig. 2a):

135 tentatively named as Iso. sp. YL-2021a and Iso. sp. YL-2021a. The former was a cryptic species (Fig. 1b) of Iso. prostoma and it had a longer body $(278 \mu \mathrm{m})$ than Iso. prostoma, while the latter was a cryptic species (Fig. 1d) of Iso. intestinalis and it had a lower ratio of

138 body length to width (1.3 on average) but longer chromosomes ( $22.5 \mathrm{~kb}$ on average) than Iso.

139 intestinalis. Additionally, the $18 \mathrm{~S}$ sequence identity between synonymic morphospecies was 
140 all $>99 \%$ while that among the four Isotrichidae species was all $<98 \%$ (Supplementary Fig.

141 6), which also supports the taxonomic revision at the species level. Accordingly, the 22

142 collected morphospecies were reclassified to 19 species.

143 The current taxonomic ranks for rumen ciliates at or above the genus level also lack

144 molecular evidence. For example, the 18S rRNA gene phylogeny placed the subfamilies

145 Entodiniinae and Ophryoscolecinae as two branching lineages within the subfamily

146 Diplodiniinae ${ }^{5,14}$. To resolve the phylogenetic challenges, we firstly constructed a maximum

147 likelihood (ML) tree using 113 concatenated single-copy proteins with Tetrahymena

148 thermophila as an outgroup (Fig. 2d). We also constructed a species tree using OrthoFinder ${ }^{28}$

149 under the coalescent model (Supplementary Fig. 7). The two trees were consistent in

150 topology and had 100\% bootstrap support for each node. The results support Entodiniinae

151 representing the oldest branch in Ophryoscolecidae and Ophryoscolecinae as a sister to

152 Diplodiniinae, which is consistent with their morphological inference ${ }^{29}$. The concatenated

153 protein-based phylogeny served as the basis for the taxonomic rank normalization at and

154 above genus using relative evolutionary divergence (RED) ${ }^{25}$. Comparing the RED value with

155 the RED intervals of taxonomic ranks (median RED \pm 0.1 ), the genus Dasytricha was

156 reassigned from Isotrichidae to a new family Dasytrichidae, which was treated as a synonymy

157 of Isotrichidae in the past ${ }^{4}$. Additionally, Ent. bursa and Ent. caudatum were reassigned to a

158 new genus Entodinium_A, and Iso. prostoma and Iso. sp. YL-2021a were reassigned to a new

159 genus Isotricha_A (Fig. 2c-d). For consistency with the family Ophryoscolecidae,

160 subfamilies Isotrichinae and Dasytrichinae were created for the families Isotrichidae and

161 Dasytrichidae, respectively. Ultimately, the 19 identified ciliate species were assigned to two

162 orders, three families, five subfamilies, and 13 genera (Fig. 2d). The genome-based

163 taxonomy supports the taxonomic worth of the size and number of skeletal plates, which is a

164 newly evolved organelle for storing amylopectin in the clades of Ophryoscolecinae and 
165 Diplodiniinae ${ }^{6}$ and useful in differentiating genera. However, the phylogenetic relationships

166

167

168

169

170

171

172

173

174

175

176

177

178

180

181

182

183

184

185

186

187

188

189

among the genera within Diplodiniinae did not reflect the number of skeletal plates, as

evidenced by Diplodinium without skeletal plate but being more closely related to

Enoploplastron with three skeletal plates than Eremoplastron with only one skeletal plate.

This suggests a single origin of the skeletal plates, which was probably lost in Diplodinium.

Invasion into the rumen was one of the most important events in the evolutionary history

of Trichostome ciliates ${ }^{5}$. As shown above, not only rumen Ophryoscolecidae, as previously

suggested $^{5}$, but also Isotrichidae have radiated in speciation. Molecular clock analyses

showed that explosive radiation of both Ophryoscolecidae and Isotrichidae occurred about 5 -

35 million years ago (Fig. 3e and Supplementary Fig. 8), which is consistent with the

duration of the rapid radiation of ruminants ${ }^{30}$, suggesting concurrent co-evolution and cospeciation between ruminants and rumen ciliates.

\section{Genome architecture and gene family diversity}

Explosive speciation can result from a series of genomic changes and innovations, and the genome catalog of the rumen ciliates provides new insights into their early diversification.

The genomic architecture features of Isotrichidae were obviously divergent from those of Dasytrichidae and Ophryoscolecidae (Supplementary Table 4), despite Dasytrichidae and Isotrichidae being in the same order. Notably, Isotrichidae had a lower GC content and longer gene-coding regions and gene introns than Dasytrichidae and Ophryoscolecidae

(Supplementary Fig. 9-10). Most interestingly, the two classic chromosome architectures of ciliates macronuclear genomes ${ }^{31}$ existed in a single subclass (Fig. 3a): the genomes of Ophryoscolecidae and Dasytrichidae were extensively fragmented (EF) with gene-sized nanochromosomes having a mean length of $2.3 \mathrm{~kb}$, while the genomes of Isotrichidae were non-extensively fragmented (NEF) minichromosomes with a mean length of $14.3 \mathrm{~kb}$, mostly encoding several genes each. The genomes of its sister-subclasses Haptoria and sister-classes 
190 Spirotrichea were both $\mathrm{EF}^{32}$, therefore, we inferred that the genome of the common ancestor

191 of rumen ciliates was EF, and the NEF genomes of Isotrichidae was an independent origin

192 according to the parsimony principle of evolution ${ }^{33}$. However, similar to EF genomes, the

193 chromosome copy numbers in the NEF genomes of Isotrichidae were nonuniform

194 (Supplementary Fig. 11), while those in the NEF genomes of the classes Heterotrichea and

195 Oligohymenpphores were approximately equal ${ }^{32}$.

196 Using OrthoFinder ${ }^{28}$, we annotated 64,189 gene families from the 52 SAGs (Fig. 3e and

197 Supplementary Fig. 10), of which, 40,898 were shared by at least two species, and 23,291

198 were identified as species-specific (ranging from 110 in Ost. mammosum to 3,818 in Oph.

199 caudatus). Of the species-shared families, only 14,871 (36.4\%) were found in both

200 Vestibuliferida and Entodiniomorphida, while the remaining gene families were

201 Vestibuliferida-specific $(6,091)$ or Entodiniomorphida-specific $(19,936)$. Of the

202 Entodiniomorphida-specific families, 10,043 arose at the clades of Diplodiniinae and

203 Ophryoscolecinae. This suggests that extensive lineage-specific gene families arose in the

204 early and during the diversification of rumen ciliates into modern orders. Additionally, compared with the common ancestor of rumen ciliates, more subfamily-shared gene families expanded in Entodiniomorphida $(2,784 / 7,031)$ than in Vestibuliferida $(53 / 7,031)$ (Fig. 3e). As a result, species in Entodiniomorphida, in particular in the clade of Diplodiniinae and Ophryoscolecinae, had larger and more diverse gene families than the species in

209 Vestibuliferida (Fig. 3d), which might have further induced the functional divergence across

210 orders and subfamilies (Fig. 3c). Comparing the differential investment in KEGG pathways

211 and Gene Ontology terms across those clades (Supplementary Fig. 12-13), we found that the

212 members of the Isotrichidae possessed a higher proportion of genes related to organismal

213 systems and environmental adaptation (e.g., endocrine, immune, detoxification, and

214 antioxidant activity), while those of Ophryoscolecidae were primarily related to biomass 
215 (e.g., carbohydrates, lipids, and xenobiotics) degradation and metabolism. Indeed, compared

216 the domains of order-specific genes between Isotrichidae and Ophryoscolecidae, Isotrichidae

217 had a relative higher predominance of HSP70 and WD40, which are related to stress

218 resistance $^{34}$ and biogenesis of motile cilia ${ }^{35}$, respectively, while Ophryoscolecidae had a

219 higher relative predominance of CAZymes-related domains (GT, GH, CBM and cellulase)

220 (Fig. 3e).

\section{Rumen ciliates possess a broad array of CAZymes}

222 The gut microbes are of huge industrial interest due to their ability to release fermentable sugars from lignocellulose $\mathrm{e}^{36-38}$. Here, we found that the rumen ciliates were also a rich source of biomass-degrading enzymes. A total of 33,693 non-redundant ( $<99 \%$ identity) CAZymes were predicted in the 52 SAGs (Fig. 4a), and only 357 had a highly similar match ( $\geq 95 \%$ identity) in any of the public protein databases (nr, env_nr, m5nr, UniProt TrEMBL) and the rumen bacterial and fungal protein datasets ${ }^{1,2,39,40}$, indicating that $99 \%$ of the CAZymes can be considered new. In particular, the species of Diplodiniinae and Ophryoscolecinae encoded as many CAZyme as gut fungi, the latter of which possesses the largest number of CAZyme genes yet known in nature ${ }^{37}$. And the species of Diplodiniinae and Ophryoscolecinae encoded more CAZyme genes than Entodiniinae (by two-fold) and Isotrichidae and Dasytrichidae (by five-fold) (Fig. 4b) with clear profile-divergences (Fig. 4c), largely attributed to the extensive gene family arising and expansion.

Predicting the substrates of glycoside hydrolases (GH) and polysaccharide lyases (PL) by categorizing their corresponding EC number (EC 3.2.1.- or EC 4.2.2.-), we found that each species of the rumen ciliates possessed a broad array of enzymes to synergistically degrade plant cell wall (cellulose, hemicellulose and pectin), plant non-structural carbohydrates (fructan and starch) and microbial carbohydrates (chitin and peptidoglycan) (Fig. 4d). 
240 polysaccharides and the resulting oligosaccharides. For example, the Ophryoscolecid SAGs

241 possessed endo- $\beta$-1,4-glucanase (EC 3.2.1.4), $\beta$-glucosidase (EC 3.2.1.21), endo-1,6- $\beta$ -

242 glucosidase (EC 3.2.1.75), and $\beta$-1,4-cellobiosidase (EC 3.2.1.91), allowing them to degrade

243 cellulose. Less than one-quarter of the degradative CAZymes (ranging from $6 \%$ in

244 Ophryoscolecinae to $24 \%$ in Isotrichinae) of ciliates were invested in degrading microbial

245 carbohydrates, suggesting that their energy acquisition through microbe predation is limited.

246 Most of the degradative CAZymes of Diplodiniinae (72\%) and Ophryoscolecinae (82\%) were

247 invested in degrading plant cell wall, while those of Isotrichinae (45\%), Dasytrichinae (44\%),

248 and Entodiniinae (44\%) were mainly invested in degrading plant non-structural carbohydrates

249 (Fig. 4e). These observations revealed the molecular mechanisms underlying the variable

250 substrate preferences across subfamilies ${ }^{6}$ and why ciliates could reach up to half of the rumen

251 microbial biomass ${ }^{6}$. The variable investment tradeoffs in degradative CAZymes partly avoid

252 an overlapping niche across subfamilies, and thus enabling a robust performance of the

253 overall biomass degradation process ${ }^{41}$. Additionally, rumen ciliates ingest substrates into

254 digestive vacuoles (Supplementary Fig. 14) to which they deliver their degradative

255 enzymes, which can help increase the binding efficiency between degrading enzymes and

256 substrates due to close proximity and improve the utilization efficiency of the degradation

257 products. Taken together, the broad array of CAZymes, variable substrate preferences, and

258 intra-vacuoles digestion in rumen ciliates may contribute to their energy acquisition and use

259 from recalcitrant plant materials and allow for their ecological success in the rumen

260 ecosystem.

261 Compared with rumen ciliates, non-gut Alveolate species $(n=13)$ possess a much smaller

262 number and lower diversity of degradative CAZymes, and they are incapable of digesting

263 xylan-related hemicellulose (xylan, xyloglucan, and arabinoxylan) or pectin (Fig. 4d),

264 suggesting that most degradative CAZymes of rumen ciliates were not likely obtained by 
vertical inheritance. To determine whether this innovation is the result of inter-kingdom horizontal gene transfer (HGT), we extracted and built phylogenetic trees of homologous sequences from the genomes of rumen bacteria, gut fungi, non-gut Alveolate, and rumen ciliates. The results showed that the CAZymes of rumen ciliates mainly originated via HGT

269 from gut bacteria and fungi (as high amino acid sequence similarity and phylogenetic nesting), and most of them had more than one HGT event, which could occur in the early or during the diversification of rumen ciliates (Fig. $4 f$ and Supplementary Fig. 15). For example, xylanase (EC 3.2.1.8) has recruited five gene families (GH5, GH10, GH11, GH30 and GH43) from bacterial and fungal donors, involving at least 15 HGT events (9 from Firmicutes, 4 from Bacteroidota, 1 from Fibrobacteria, and 1 from fungi). between ciliates and the corresponding donors, such as the loss of the CBM22 domain in EC above four enzymes (Fig. 4g and Supplementary Fig. 16). To investigate the enzyme

280 activities differences between horizontally transferred CAZymes, we cloned and overexpressed one cellulase (EC 3.2.1.4 in GH5) and one xylanase (EC 3.2.1.8 in GH10) cellulase and xylanase had nine-fold and two-fold higher activity than those of bacterial donors, respectively (Fig. 4h).

Additionally, $25.6 \%$ of the degradative CAZymes could not be annotated to any EC numbers. We evaluated the substrates and activity of one GH19 protein of Oph. caudatus also

287 after cloning and overexpression in P. pastoris, and the results showed that it was a lysozyme (with a specific activity of $36,007 \mathrm{U} / \mathrm{mg}$ ), and the purified GH19 protein inhibited 
290 characteristic selective inhibition of the Gram-positive bacterium suggests that this ciliate

291 lysozyme was a potential alternative to monensin, which is one of the most successful rumen

292 modifiers in improving ruminant feed efficiency but has been banned in some countries

293 because it is an ionophore and appears residues in milk and meat ${ }^{42}$.

\section{Enabling metagenomic analyses of rumen ciliates}

295 The sequenced genomes can greatly facilitate metagenomic analyses of microbiomes including communities of rumen ciliates. We re-analyzed 902 publicly available rumen metagenomes (Supplementary Table 3) against three datasets: 1) the RefSeq genomes and rumen microbial genomes ${ }^{2,43}, 2$ ) dataset 1) plus the SAGs of the rumen ciliates, and 3) dataset 2) plus the metagenome-assembled ciliate sequences. The results showed that the read

300 classification rates of metagenome as rumen ciliates could be affected by the host, diet, and management (Fig 5a, c). Of note, the classification rates as rumen ciliates decreased by $99 \%$ in the metagenomes of sheep that suffered from subacute ruminal acidosis due to feeding of a high concentrate $\operatorname{diet}^{44}$, which is consistent with previous experimental findings that ciliates are sensitive to $\mathrm{pH}^{45}$. Of the 902 metagenomes, 726 were from animals fed a diet with a concentrate content of $<80 \%$, which is considered a low risk for subacute ruminal acidosis according to the dramatic drop of ciliate abundance. And in the 726 rumen metagenomes, our

307 ciliate dataset allowed for classification of $12 \%$ of the total reads on average, with a large range from $0.1 \%$ to $72 \%$ (Fig 5a). With the improvement of ciliate dataset, the total read

309 classification rates across metagenomes increased and became more convergent than

310 otherwise. For example, with the improvement of ciliate dataset, the total read classification

311 rates in the 285 samples of Stewart et al. ${ }^{2,38}$ increased from $39-92 \%$ to $72-94 \%$, and one-fold

312 more of the samples (33\% vs. $73 \%$ ) achieved a classification rate of $80 \%$ or higher (Fig $\mathbf{5 b}$ ).

313 Of the identified ciliate reads in the metagenomes, more than $73 \%$ could be classified based 314 on SAGs, suggesting that the collected ciliates comprised a considerable fraction of the 
315 rumen ciliates community (Fig 5d). Interestingly, based on microscopic counting,

316 Entodiniinae as the most dominant rumen ciliates can account for $>90 \%$ of the total rumen

317 ciliates in domesticated ruminants (Supplementary Table 2), but only $\sim 25 \%$ of the identified

318 ciliate sequence reads were assigned to Entodiniinae. This discrepancy might be attributable

319 to (1) the lack of the genomes of some abundant Entodiniinae species, such as Ent. dilobum

320 and Ent. rostratum, and (2) the ploidy of Entodiniinae was likely lower than that of other

321 rumen ciliates.

\section{Discussion}

323 Compared with gut bacteria and $\operatorname{archaea}^{1-3}$, eukaryotic microbes (protozoa and fungi) are understudies and represent an underexplored genetic resource ${ }^{46}$. Here, we used single-cell sequencing to circumvent the lack of the inability to establish axenic cultures of rumen ciliates and developed a ciliate-telomere-capped sequence dataset to aid the identification of ciliate sequences from mixed sequences. Compared with previous studies on fungi and

328 flagellate ${ }^{47,48}$, the more successfully captured ciliate genomes from individual cells were largely attributed to their mini-/nano-chromosomes, which typically have a high ploidy ${ }^{20,24}$. Metagenomic binning is the other technique to recover uncultured microbial genomes ${ }^{36}$, but it is not applicable to recover ciliate genomes due to their large number and nonuniform copy numbers of chromosomes and the dependence of binning on sequencing depth. In this study, the unparalleled genome catalog of rumen ciliates shines new lights on their taxonomy, evolution, and metabolism, and also greatly facilitates the rumen metagenomic analyses by allowing $\sim 12 \%$ of reads to be classified. including ciliates is challenging ${ }^{25,26}$ because the reproductive isolation principles were not or mostly not applicable and pure cultures are not available for most microorganisms. 
340 for rumen ciliates, which allowed $45 \%$ of the 22 collected morphospecies to be changed to

341 their existing taxonomy. Using this revised taxonomy, nine synonymic Ophryoscolecidae

342 morphospecies, two cryptic Isotrichidae species and two new genera were identified, and

343 genus Dasytricha was reassigned from Isotrichidae to a new family Dasytrichidae. The

344 genome-based taxonomy corroborated or rejected the taxonomic worth of some

345 morphological features of rumen ciliates, which will help to revise the morphology-based

346 taxonomy and to identify new species.

347 Additionally, we provided a phylogenetic backbone to understand the evolutionary

348 processes of specific traits and early diversification in rumen ciliates, such as the origin of the

349 non-extensively fragmented minichromosomes in Isotrichidae, the origin and loss of skeletal

350 plates in Ophryoscolecidae. The identified novel traits will facilitate future research on

351 ciliates in general. For example, compared with the previous inter-class investigations ${ }^{49,50}$, the

352 rumen ciliates will provide a more allied model for investigating the unsolved molecular

353 mechanisms underlying chromosome fragmentation ${ }^{21}$.

354 Importantly, we showed that rumen ciliates were another important weapon for

355 ruminants to degrade nearly all kinds of plant-derived polysaccharides. In particular, the

356 species of Diplodiniinae and Ophryoscolecinae encoded as many CAZyme genes as gut

357 fungi, and $\sim 80 \%$ of their degrading enzymes acted on plant cell wall. Most of the ciliate

358 degradative CAZymes were obtained via HGT from bacteria and fungi ${ }^{7}, 17$, but they

359 underwent considerable sequence divergence from the donor. The experimentally identified

360 promising activity of their cellulase, xylanase and lysozyme reflects the potential application

361 of ciliate CAZymes in biomass conversion and microbiota modulation.

\section{Methods}

363 Single-ciliate-cell isolation, identification and sequencing

364 All the experimental procedures on animals were conducted per the guidelines of the 
365 Regulations for the Administration of Affairs Concerning Experimental Animals (Ministry of

366 Science and Technology, China, 2013) and were approved by the Northwest A\&F University

367 Animal Care and Use Committee.

368 Individual ciliate cells were isolated from fresh rumen fluid samples collected from 15

369 ruminally fistulated ruminants (6 Holstein cattle, 3 Qinchuan beef steers, and 6 Guanzhong

370 dairy goats) all kept in Yangling, Shannxi Province, China using glass micropipettes under an

371 inverted microscope (Nikon, TI-FL, Japan). We purposely selected ciliates with distinct

372 morphologies. The single ciliate cells were repeatedly transferred and washed using MB9

373 buffer $^{51}$ until no other ciliate cells could be found microscopically. The washed single ciliate

374 cells were then transferred to individual microtubes each containing $2 \mu 1$ of cell lysis buffer. A

375 total of 69 single ciliate cells representing 22 morphospecies (as identified morphologically,

376 detailed below) were isolated, and 45 of them were subjected to whole-genome amplification

377 (WGA) using multiple displacement amplification ${ }^{52}$ and the remaining 24 single ciliate cells

378 were subjected to parallel WGA and whole-transcriptome amplification (WTA) using the

379 G\&T-seq ${ }^{22,53}$ (Supplementary Table 1). Illumina TruSeq libraries were prepared for each of

380 the amplified genomes and transcriptomes and then sequenced $(2 \times 150 \mathrm{bp})$ on an Illumina

381 NovaSeq platform.

382 The morphological identification of rumen ciliates ${ }^{54}$ was conducted with light

383 microscopy, scanning electron microscopy, and confocal laser scanning microscopy. The

384 morphological description and micrographs of the isolated ciliate species are summarized in

385 Supplementary Fig. 1-3. The taxonomic identification of the collected single ciliates was

386 confirmed by sequencing analysis of their 18S rRNA genes after the genome assembly. The

387 ciliates in the rumen samples were counted using a Sedgewick-Rafter chamber as described

388 previously ${ }^{54}$

389 Genome assembly and ciliate sequence identification 
390 The cleaned Illumina reads of each WGA sequencing were assembled using both MEGAHIT

$391 \quad(\mathrm{v} 1.2 .1)^{55}$ and SPAdes (v3.13.1 $)^{56}$. The contigs assembled by the two assemblers were merged

392 into longer contigs using quickmerge $(\mathrm{v} 1.2 .1)^{57}$.

393 The sequence characteristics of rumen ciliates were identified as follows. Firstly, the

394 ends (30 bp) of WGA contigs were used to identify telomeric repeats using MEME

$395(\mathrm{v} 4.12 .0)^{58}$, and the telomeric repeats of the collected morphospecies were all identified as

396 (5'-CCCCAAT)n. Secondly, complete chromosomes (contigs that were capped with at least

3971.5 telomeric repeats without mismatch at both ends) were extracted from the WGA contigs

398 using a python script ${ }^{32}$. Contigs with potential contamination sequences (each with a $>20 \%$

399 GC content for the subtelomeric region (100 bp immediately adjacent to the telomere) and a

$400 \mathrm{GC}$ content $>44 \%$ GC for the rest of the contig) were filtered out with an error rate of $1 \%$

401 allowed.

402 The publicly available rumen metagenomes ( $\mathrm{n}=902$, Supplementary Table 3) were

403 downloaded from the NCBI SRA, and were assembled using MEGAHIT. These contigs were

404 also subjected to decontamination as mentioned above. The decontaminated contigs from the

405 SAGs and the metagenome-assembled contigs were combined into an $18 \mathrm{~Gb}$ sequence dataset

406 of rumen ciliate macronuclear sequences, which were capped with telomeric repeats at one or

407 both ends (Supplementary Fig. 4). The $18 \mathrm{~Gb}$ of contigs of ciliates plus other rumen

408 microbial genomes $2,36,38-40,43,59,60$ were used as a reference to pick the ciliate sequences that

409 do not have any telomeres in the WGA assembled sequences using blastn (v2.6.0+). The

410 sequences with the best match to ciliate sequences ( $>85 \%$ identity over $>500$ bp and $>50 \%$

411 coverage of reference or query) were considered as ciliate sequences.

412 The telomereless contigs picked above and the telomere-capped contigs with $>3 \mathrm{X}$ of read

413 depth were retained in SAGs. Genome completeness of each SAG was assessed using

414 BUSCO v $5^{61}$ with OrthoDB v10 $10^{23}$ using the predicted protein-coding genes as input. Of the 
41569 SAGs (Supplementary Table 4), $52 \mathrm{had} \geq 80 \%$ of the 171 Alveolata conserved marker

416 genes and were considered "high-quality", while 17 had $<80 \%$ of the Alveolata conserved

417 marker genes.

\section{Gene prediction}

419 The ciliates in rumen fluid samples $(\mathrm{n}=14)$ were enriched by filtering $(150 \mu \mathrm{m})$ and

420 centrifugation $(500 \times \mathrm{g}$ for $5 \mathrm{~min})$. The eukaryotic mRNAs in the ciliate-enriched samples

421 were extracted and purified as previously described ${ }^{61}$, and used for metatranscriptome

422 sequencing. The single-cell transcriptomic and metatranscriptomic sequencing reads were mapped to SAGs using Hisat2, and assigned to each species.

Protein-coding genes were de novo annotated using AUGUSTUS (v3.2.3) ${ }^{62}$. Firstly, the WTA sequence reads were assembled using Trinity $(\mathrm{v} 2.8 .4)^{63}$. The assembled transcripts were used to train the de novo gene prediction model ${ }^{62}$. The best model was that of Pol. multivesiculatum with $93.5 \%$ sensitivity and $85.7 \%$ specificity at the exon level. Gene predictions of SAGs were performed also using AUGUSTUS and the best model with mapped RNA-seq data as "hints". The tRNAs-coding and rRNA genes were identified using tRNAscan-SE (v2.0.5) and Barrnap (v0.9), respectively.

\section{Circumscribing species}

The ANI and alignment coverage between genomes were calculated using pyani (v0.2.10) ${ }^{64}$ with the default parameters. A clear gap existed between $92 \%$ and $97 \%$ in ANI values, and thus ANI $>97 \%$ was considered synonym, and ANI $<92 \%$ was inter-species circumscription.

\section{Phylogenomic tree construction and higher taxonomic rank normalization}

A total of 113 single-copy homologous genes were identified among the 19 representative genomes and Tet. thermophila genomes (as outgroup) by OrthoFinder (v2.5.2) ${ }^{28}$. These single-copy genes were aligned and concatenated into supergenes for ML-based phylogenetic using RAxML (v.8.2.9) ${ }^{65}$. A species tree was also constructed using OrthoFinder based on the 
52 SAGs.

The concatenated protein-based phylogeny of the 19 rumen ciliates species served as the basis for high taxonomic rank (at and above genus) normalization using the RED values calculated with PhyloRank (v0.1.0 $)^{25}$. The RED intervals for normalizing taxonomic ranks were defined as the median RED value for taxa at each rank \pm 0.1 . Comparing the RED values with the RED intervals of taxonomic ranks, the taxa that fell outside of their RED distribution was corrected.

\section{Divergence time estimation and gene family expansion}

Divergence times of these species were estimated based on the ML tree via Bayesian relaxed molecular clock approach using MCMCtree program in the PAML package (v.4.9) ${ }^{66}$. The calibrated points of the common ancestor of rumen ciliate species $(<135 \mathrm{Mya})$ and ophryoscolecid species ( $<55$ Mya) were obtained from Vd’ačný et al. ${ }^{5}$.

Orthologs gene families among the 52 SAGs were identified using the OrthoFinder. To reduce the limitation of single-cell genomes, the maximum gene number in each gene family among different SAGs of each species was used in the CAFE (v5.0) ${ }^{67}$ to infer the expansion and contraction of gene families.

\section{Functional annotation}

Functional annotation (KEGG, GO and $\mathrm{COG}$ ) of the predicted proteins was performed using eggNOG mapper (v2.0.1) with the DIAMOND mapping mode, based on the eggNOG 5.0 orthology data ${ }^{68}$. The protein domain was annotated using pfam_scan.pl compared to Pfam_A.CAZymes annotation was executed using dbCAN $2{ }^{69}$ against the CAZyme database V9. The CAZymes were categorized with their corresponding EC number by aligning against uniprot_sprot and CAZyme database V9 using Diamond (v0.9.21.122) with an $E$-value cutoff of e-3.

\section{Identification of CAZymes with HGT signature}


465 To compare the CAZyme numbers and profiles of rumen ciliates with rumen bacteria $(\mathrm{n}=$ 466 2,405), gut fungi $(\mathrm{n}=8)$, non-gut Alveolate $(\mathrm{n}=13)$ (Supplementary Table 5), the CAZymes

467 of the latter microbes were also annotated as above. The 2,405 rumen bacterial genomes 468 served as the representative genome at species-level (ANI $>95 \%$ ) for the 4,941 metagenome469 assembled genomes from Stewart et al. ${ }^{2}$ and 410 genomes from the Hungate 1000 project $^{1}$.

$470 \quad$ For each CAZyme (the same family with the same EC number), a phylogenetic tree among rumen ciliates and other microbes was constructed using IQ-TREE (v2.1.4-beta) ${ }^{70}$ with 100 bootstrap replicates. The potential HGT events were inferred using the pipeline and cut-offs of Haitjema et al. ${ }^{39}$.

\section{Catalytic activity assay of cellulase and xylanase}

475 In vitro enzymatic activity assays were used to test the the cellulase (EC 3.2.1.4 in GH5) from rumen ciliate (Oph. caudatus) and its HGT donor (Treponema bryantii NK4A124), and xylanase (EC 3.2.1.8 in GH10) from rumen ciliate (Ost. gracile) and its HGT donor (Ruminococcus flavefaciens YRD2003). Four genes were codon-optimized according to $P$. pastoris preference. Construction of recombinant plasmids and heterologous expression and 480 purification followed the procedures previously described ${ }^{71}$. The cellulase and xylanase activities were measured as previously described ${ }^{72,73}$. Each experiment was performed with three reaction replicates to determine the mean \pm s.d. of the catalytic activity value of the enzymes.

\section{Catalytic activity assay of lysozyme}

485 The substrates and activity of one GH19 (it includes lysozyme and chitinase) protein of $O p h$. caudatus were examined after cloning into P. pastoris. The enzymatic substrate assay showed

487 that it was a lysozyme, which activity were measured as previously described ${ }^{74}$. The 
bacteria, respectively, zone assay on agarose plates.

491 Sequencing reads classification

492 The sequencing reads of 902 publicly available rumen metagenomes were classified using

493 Kraken (v2.0.7-beta) ${ }^{75}$ against three custom databases: 1) Public database, consisting of all

494 microbial genomes from RefSeq (release 201) plus rumen microbial genomes; 2)

495 Public + SAGs database, 3) Public+Ciliates database, consisting of public database, all 52

496 SAGs, and the ciliate-telomere-capped sequences from the 902 metagenomes.

\section{Data availability}

498 All sequencing data and assembled genomes have been deposited in the NCBI database with

499 accession ID PRJNA777442. All other relevant data are available in this article and its

500 Supplementary Information files, or from the corresponding author upon request.

501 Acknowledgements

502 This study was financially supported by the National Natural Science Foundation of China

503 (U21A20247, 31822052, to Y.J., and 31902126, to Z.L.) and the China Postdoctoral Science

504 Foundation (2019M663841, to Z.L.). We thank the High-Performance Computing Platform

505 of Northwest A\&F University for providing computing resources.

\section{Author contributions}

507 Z.L. and Y.J. conceived and supervised the project. Z.L., X.W., T.Z., Y.L., T.S. and X.X

508 collected the samples. X.W., Z.L., Y.L., F.L., Y.H., H.H., J.N., and J.T. carried out the

509 experiments. Z.L., Y.Z., X.W., T.Z., X.D., X.P., R.J., Y.Y., and S.G. performed bioinformatic

510 analyses. Z.L. wrote the manuscript. Z.Y., Y.J., H.H., J.Y. and B.Y. revised the manuscript. All

511 authors have read and approved the final manuscript.

\section{Competing interests}

513 The authors declare no competing interests. 


\section{Figure legend}

516 Fig. 1. Light micrographs of 22 rumen ciliate morphospecies and two cryptic species

517 examined in this study, and a brief pipeline for recovering single-ciliate genomes. a, Iso.

518 prostoma. c, Iso. intestinalis. e, Das. ruminantium. f, Ent. longinucleatum. g, Ent. bursa. h,

519 Ent. caudatum. i, Dip. anisacanthum. j, Dip. dentatum. k, Dip. flabellum monospinatum. $\mathbf{1}$,

520 Dip. flabellum aspinatum. m, Eno. triloricatum. n, Met. minomm. o, Ere. rostratum. p, Ost.

521 gracile. q, Ost. venustum. r, Ost. mammosum. s, Pol. multivesiculatum. t, Epi. caudatum. u,

522 Epi. cattanei. v, Oph. bicinctus. w, Oph. caudatus. x, Oph. purkynjei. Iso. sp. YL-2021a (b)

523 and Iso. sp. YL-2021b (d) were two cryptic species of Isotrichidae. Micrographs $\mathbf{n}$ and $\mathbf{p}$

524 show stained skeletal plates. Scale bars, $50 \mu \mathrm{m}(\mathrm{a}-\mathrm{x})$. More details of the rumen ciliates are

525 shown in Supplementary Fig. 1.

526

527 Fig. 2. Genome-based taxonomy and phylogeny of rumen ciliates. a, A heatmap showing

528 the ANI among the 52 SAGs and the scanning electron microscopy images of 22

529 corresponding morphospecies and two cryptic species. b, ANI distributions. c, Rank

530 normalization through relative evolutionary divergence (RED) of taxa. The red dot represents

531 rank reassignment. The RED interval for each rank is shown by two vertical black lines,

532 median \pm 0.1 . d, The maximum likelihood phylogenetic tree of 19 rumen ciliate species and $T$.

533 thermophila (as the outgroup) based on 113 concatenated single-copy proteins. All nodes

534 have $100 \%$ bootstrap support. The black asterisks indicate the two cryptic species identified

535 in this study.

536 Fig. 3. Genome characteristics of rumen ciliates. a Chromosome length (the red triangles

537 indicate N50) and $\mathbf{b}$ genome size distributions across five subfamilies. c Functional

538 divergence based on the clans of Pfam (P-value of Adonis $<0.001)$. d Rank curves showing

539 gene number divergence in the subfamilies-shared gene families. e Gene family divergence: 
540 From left to right: a time phylogenetic tree of 19 rumen ciliate species with gene family

541 expansions and contractions; comparison of the gene family repertoires among the 19 ciliate

542 species; a heat map showing the top 10 categories of domains found in order-specific gene

543 families of each species.

544 Fig. 4. CAZyme profiles of rumen ciliates. a, Max similarities (amino acid sequences) of

545 the CAZymes identified in the rumen ciliates compared to the public databases for seven

546 classes of CAZymes. GH, glycoside hydrolase; GT, glycosyl transferase; PL, polysaccharide

547 lyases; CE carbohydrate esterases; AA, auxiliary activities; CBM, carbohydrate-binding

548 modules, and SLH, S-layer homology modules. The numeric values in parentheses refer to

549 the numbers of genes of each class. b, Number of CAZyme-coding genes in the

550 representative genomes of five rumen ciliate subfamilies and gut fungi. c, A PCoA plot

551 showing the CAZyme profiles across the five subfamilies (the color schemes are the same as

552 in Fig 4b), and the P-value of Adonis is $<0.001$. d-e, The mean number and prevalence of

553 degradative CAZymes encoded in the genomes of rumen bacteria $(n=2,045)$, gut fungi

$554(\mathrm{n}=8)$, non-gut Alveolate $(\mathrm{n}=13)$, and the five subfamilies of rumen ciliates $(\mathrm{n}=19)$. $\mathbf{f}, \mathrm{An}$

555 example for the xylanase (EC 3.2.1.8_GH10) of rumen ciliates originated via horizontal gene

556 transfer (HGT) from rumen bacteria. $\mathbf{g - h}$, Structural and activity divergences between rumen

557 ciliates and its bacterial donors for cellulase and xylanase. The symbols *** represent a

558 statistical significance of P-values $<0.001$. i, Inhibition zone assays of a ciliate lysozyme in

559 GH19.

560 Fig. 5. Metagenomic analyses. a, The read classification rates as ciliates across rumen

561 metagenomes of different hosts fed a diet with $<80 \%$ concentrate; $\mathbf{b}$, Improvement of

562 classification rates of total reads in two previous studies ${ }^{2,76}$ enabled by the ciliate dataset; c,

563 The association of read classification rates as rumen ciliates with diet and management ${ }^{44,77}$.

564 Statistical significance was determined by the two-side Wilcoxon rank-sum test. Sheep were 
565 fed diets with low-level concentrate $(\mathrm{CON}$, control, $\mathrm{n}=8)$ or high-level concentrate that

566 induced subacute ruminal acidosis (SARA, $n=8)^{44}$. Holstein dairy cows were house fed a corn

567 silage and concentrate $\operatorname{diet}(n=23)$ or grazed on a natural prairie $(n=20)^{77}$. d, Relative

568 abundance of rumen ciliates.

\section{Supplementary figure legend}

571 Supplementary Fig. 1. Morphological description and micrographs of the 19 studied rumen

572 ciliate species including the identified synonymic and cryptic species. The micrographs from scanning electron microscopy, light microscopy, and confocal laser scanning microscopy; and the cited schematic diagram ${ }^{78}$ and morphological description ${ }^{6}$ are presented. The skeletal plate was stained by Lugo's iodine. Scale bars, $50 \mu \mathrm{m}(\mathrm{a}-\mathrm{x})$.

Supplementary Fig. 2. Summary of rumen ciliates isolated from host animals (a) and

577 genome-based subfamilies and genera (b).

578 Supplementary Fig. 3. The difference in secondary caudal spines between morphospecies Oph. caudatus (three circles) and Oph. bicinctus (two circles).

Supplementary Fig. 4. A pipeline for recovering single-ciliate genomes and gene prediction.

The original array consists of three parts: public metagenomic data, metatranscriptomic data, and single-ciliate genomic and transcriptomic data from whole-genome and wholetranscriptome amplification sequencing.

584 Supplementary Fig. 5. Comparison of genomes characteristics by different sequencing depth and assembly strategies. a-d, Contig N50, genome size, the ratio of telomere-capped contigs using different sequencing data size when the genomes were assembled by Megahit. e-f.

587 Contig N50 and genome size, the ratio of telomere-capped contigs when the genomes were assembled by different assembly strategies: single software (Megahit, Spades) or hybrid assembly (Cd-hit and Quickmerge). Average size ratio of telomere-capped contigs in 
590 Isotrichidae (g) and Ophryoscolecidae genomes (h) with different assembly strategies.

591 Supplementary Fig. 6. a Pairwise average nucleotide identity (ANI) and coverage

592 comparison calculated for the 52 SAGs. b Histogram of pairwise ANI values of

593 morphospecies. c Pairwise nucleotide identity of 18S rRNA gene across SAGs.

594 Supplementary Fig. 7. Species tree of 52 rumen single-ciliate genomes inferred using

595 OrthoFinder with the coalescent model.

596 Supplementary Fig. 8. Divergence time estimates within rumen ciliates inferred by

597 MCMCtree. The estimated divergence times with 95\% credibility intervals are labeled on

598 each node.

599 Supplementary Fig. 9. Key features of protein-coding chromosomes of Isotrichidae and

600 Ophryoscolecidae, using Isotricha sp. YL-2021b and Polyplastron multivesiculatum as

601 examples, respectively. Representative chromosome features are shown on average length.

602 UTR, untranslated region; UTS, untranscribed region.

603 Supplementary Fig. 10. Genome characteristics of rumen ciliates. Gene intron length (a)

604 and genomic GC content (b), gene family richness (c) and profiles (d) across five

605 subfamilies. e Number of gene families along with an additional SAG. $\mathbf{f}$ Comparison of the

606 gene repertoires of 19 ciliate species. $g$ The shared and unique gene families in the five

607 subfamilies of rumen ciliates.

608 Supplementary Fig. 11. The relative chromosome copy number distribution in rumen ciliate

609 genomes.

610 Supplementary Fig. 12 Comparison of the KEGG pathway repertoires of rumen ciliates.

611 Significant differences among subfamilies were assessed with Fisher's exact test. ${ }^{\mathrm{a}-\mathrm{d}}$ Means

612 with different superscripts within a row differ $(\mathrm{P}<0.05)$. 
613 Supplementary Fig. 13 Comparison of the Gene Ontology repertoires of rumen ciliates.

614 Significant differences among subfamilies were assessed with Fisher's exact test. ${ }^{\mathrm{a}-\mathrm{d}}$ Means

615 with different superscripts within a row differ $(\mathrm{P}<0.05)$.

616 Supplementary Fig. 14 Photomicrograph of rumen ciliates were engulfing a plant fiber

617 Supplementary Fig. 15 Examples for the CAZymes of rumen ciliates originated via

618 horizontal gene transfer from gut bacteria and fungi or via vertical inheritance from non-gut

619 Alveolate.

620 Supplementary Fig. 16 Examples for structural divergences of horizontally transferred

621 CAZymes between ciliates and the corresponding donors

622

623 Supplementary Table 1 Background information on the single-ciliate-cell samples.

624 Supplementary Table 2 Relative abundances of rumen ciliates across our collected samples

625 and published studies as evaluated by microscopic counting.

626 Supplementary Table 3 Characteristics and sources of rumen metagenomic datasets used in 627 this study.

628 Supplementary Table 4 The statistic information of the single-ciliate-cell amplified 629 genomes.

630 Supplementary Table 4 The statistic information of the single-ciliate-cell amplified

631 genomes.

632 Supplementary Table 5 The genomes of gut fungi $(n=8)$ and non-gut Alveolate $(n=13)$ used 633 in this study

\section{Reference}

636 1. Seshadri, R. et al. Cultivation and sequencing of rumen microbiome members from the 
638 2. Stewart, R. D. et al. Compendium of 4,941 rumen metagenome-assembled genomes for 639 rumen microbiome biology and enzyme discovery. Nat. Biotechnol. 37, 953-961 (2019).

640 3. Almeida, A. et al. A unified catalog of 204,938 reference genomes from the human gut microbiome. Nat. Biotechnol. 1-10 (2020) doi:10.1038/s41587-020-0603-3.

4. Lynn (eds.), D. H. The Ciliated Protozoa: Characterization, Classification, and Guide to the Literature. (Springer Netherlands, 2008).

5. Vd'ačný, P. Evolutionary Associations of Endosymbiotic Ciliates Shed Light on the Timing of the Marsupial-Placental Split. Mol. Biol. Evol. 35, 1757-1769 (2018).

6. Williams, A. G. \& Coleman, G. S. The Rumen Protozoa. (Springer-Verlag New York, 1992).

7. Park, T., Wijeratne, S., Meulia, T., Firkins, J. L. \& Yu, Z. The macronuclear genome of anaerobic ciliate Entodinium caudatum reveals its biological features adapted to the distinct rumen environment. Genomics 113, 1416-1427 (2021).

8. Gruby, D. \& Delafond, H. Recherchessur des animalcules se devéloppant en grand nombredans $1^{`}$ estomacetdan les intestins pendant la digestion des animaux herbivores et carnivores. C R Acad Sci Paris 17, 1304-1308 (1843).

9. Russell, J. B. Factors That Alter Rumen Microbial Ecology. Science 292, 1119-1122 (2001).

10. Firkins, J. L. Extending Burk Dehority's Perspectives on the Role of Ciliate Protozoa in the Rumen. Front. Microbiol. 11, 17 (2020).

11. Hobson, P. N. \& Stewart, C. S. The Rumen Microbial Ecosystem. (Springer Netherlands, 1997).

12. Dehority, B. A. Rumen Ciliate Protozoa of the Blue Duiker (Cephalophus monticola), with Observations on Morphological Variation Lines Within the Species Entodinium dubardi. J. Eukaryot. Microbiol. 41, 103-111 (1994). 
663 13. Tymensen, L., Barkley, C. \& McAllister, T. A. Relative diversity and community 664 structure analysis of rumen protozoa according to T-RFLP and microscopic methods. $J$. 665 Microbiol. Methods 88, 1-6 (2012).

666 14. Moon-van der Staay, S. Y. et al. The symbiotic intestinal ciliates and the evolution of 667 their hosts. Eur. J. Protistol. 50, 166-173 (2014).

668 15. Zhao, Y. et al. Utility of combining morphological characters, nuclear and mitochondrial genes: An attempt to resolve the conflicts of species identification for ciliated protists. Mol. Phylogenet. Evol. 94, 718-729 (2016).

16. Li, Z. et al. Dynamics of methanogenesis, ruminal fermentation and fiber digestibility in ruminants following elimination of protozoa: a meta-analysis. J. Anim. Sci. Biotechnol. 9, (2018).

17. Feng, J.-M. et al. Single-cell transcriptome sequencing of rumen ciliates provides insight into their molecular adaptations to the anaerobic and carbohydrate-rich rumen microenvironment. Mol. Phylogenet. Evol. 143, 106687 (2020).

18. Blackburn, E. H. \& Gall, J. G. A tandemly repeated sequence at the termini of the extrachromosomal ribosomal RNA genes in Tetrahymena. J. Mol. Biol. 120, 33-53

679 (1978).

680

19. Greider, C. W. \& Blackburn, E. H. A telomeric sequence in the RNA of Tetrahymena telomerase required for telomere repeat synthesis. Nature 337, 331-337 (1989).

20. Swart, E. C. et al. The Oxytricha trifallax Macronuclear Genome: A Complex Eukaryotic Genome with 16,000 Tiny Chromosomes. PLoS Biol. 11, e1001473 (2013). relationship to the massively rearranged somatic genome. eLife $\mathbf{5}$, (2016). transcriptomes. Nat. Methods 12, 519-522 (2015). 
688

689

690

691

692

693

694

695

696

697

698

699

700

701

702

703

704

705

706

707

708

709

710

711

712

23. Kriventseva, E. V. et al. OrthoDB v10: sampling the diversity of animal, plant, fungal, protist, bacterial and viral genomes for evolutionary and functional annotations of orthologs. Nucleic Acids Res. 47, D807-D811 (2019).

24. Zheng, W., Wang, C., Lynch, M. \& Gao, S. The Compact Macronuclear Genome of the Ciliate Halteria grandinella: A Transcriptome-Like Genome with 23,000 Nanochromosomes. mBio 12, (2021).

25. Parks, D. H. et al. A standardized bacterial taxonomy based on genome phylogeny substantially revises the tree of life. Nat. Biotechnol. (2018) doi:10.1038/nbt.4229.

26. Parks, D. H. et al. A complete domain-to-species taxonomy for Bacteria and Archaea. Nat. Biotechnol. 1-8 (2020) doi:10.1038/s41587-020-0501-8.

27. Jain, C., Rodriguez-R, L. M., Phillippy, A. M., Konstantinidis, K. T. \& Aluru, S. High throughput ANI analysis of $90 \mathrm{~K}$ prokaryotic genomes reveals clear species boundaries. Nat. Commun. 9, (2018).

28. Emms, D. M. \& Kelly, S. OrthoFinder: solving fundamental biases in whole genome comparisons dramatically improves orthogroup inference accuracy. Genome Biol. 16, 157 (2015).

29. Rumen microbiology: from evolution to revolution. (Springer, 2015).

30. Chen, L. et al. Large-scale ruminant genome sequencing provides insights into their evolution and distinct traits. Science 364, eaav6202 (2019).

31. Prescott, D. M. The DNA of ciliated protozoa. Microbiol. Rev. 58, 233-267 (1994).

32. Maurer-Alcala, X. X., Yan, Y., Pilling, O. A., Knight, R. \& Katz, L. A. Twisted Tales: Insights into Genome Diversity of Ciliates Using Single-Cell 'Omics. Genome Biol Evol 13 (2018).

33. Hillis, D. M. \& Moritz, C. Molecular Systematics. (Molecular Systematics, 1990).

$$
\text { doi:10.1007/bf00162977. }
$$


713 34. La Terza, A., Papa, G., Miceli, C. \& Luporini, P. Divergence between two Antarctic species of the ciliate Euplotes, E-focardii and E-nobilii, in the expression of heat-shock protein 70 genes. Mol. Ecol. 10, 1061-1067 (2001).

35. Sharma, N. et al. Katanin regulates dynamics of microtubules and biogenesis of motile cilia. J. Cell Biol. 178, 1065-1079 (2007).

36. Hess, M. et al. Metagenomic Discovery of Biomass-Degrading Genes and Genomes from Cow Rumen. Science 331, 463-467 (2011).

37. Solomon, K. V. et al. Early-branching gut fungi possess a large, comprehensive array of biomass-degrading enzymes. Science 351, 1192-1195 (2016).

38. Stewart, R. D. et al. Assembly of 913 microbial genomes from metagenomic sequencing of the cow rumen. Nat. Commun. 9, 870 (2018).

39. Haitjema, C. H. et al. A parts list for fungal cellulosomes revealed by comparative genomics. Nat. Microbiol. 2, 17087 (2017).

40. Solden, L. M. et al. Interspecies cross-feeding orchestrates carbon degradation in the rumen ecosystem. Nat. Microbiol. 3, 1274 (2018).

41. Rubino, F. et al. Divergent functional isoforms drive niche specialisation for nutrient acquisition and use in rumen microbiome. ISME J. 11, 932-944 (2017).

42. Shen, J., Liu, Z., Yu, Z. \& Zhu, W. Monensin and Nisin Affect Rumen Fermentation and Microbiota differently in vitro. Front. Microbiol. 8, (2017).

43. Gharechahi, J. et al. Metagenomic analysis reveals a dynamic microbiome with diversified adaptive functions to utilize high lignocellulosic forages in the cattle rumen. ISME J. (2020) doi:10.1038/s41396-020-00837-2. Microbial Ecosystem. DNA Res. 21, 115-125 (2014). 
of High-Concentrate Feeding and Low Ruminal pH on Methanogens and Protozoa in the Rumen of Dairy Cows. Microb. Ecol. 62, 94-105 (2011).

740

46. Marmeisse, R., Kellner, H., Fraissinet-Tachet, L. \& Luis, P. Discovering Protein-Coding Genes from the Environment: Time for the Eukaryotes? Trends Biotechnol. 35, 824-835 (2017).

47. Gawryluk, R. M. R. et al. Morphological Identification and Single-Cell Genomics of Marine Diplonemids. Curr. Biol. 26, 3053-3059 (2016).

48. Ahrendt, S. R. et al. Leveraging single-cell genomics to expand the fungal tree of life. Nat. Microbiol. 3, 1417 (2018).

49. Zufall, R. A., McGrath, C. L., Muse, S. V. \& Katz, L. A. Genome Architecture Drives Protein Evolution in Ciliates. Mol. Biol. Evol. 23, 1681-1687 (2006).

50. Yan, Y., Maurer-Alcalá, X. X., Knight, R., Kosakovsky Pond, S. L. \& Katz, L. A. SingleCell Transcriptomics Reveal a Correlation between Genome Architecture and Gene Family Evolution in Ciliates. mBio 10, e02524-19, /mbio/10/6/mBio.02524-19.atom (2019).

51. Or-Rashid, M. M., Odongo, N. E. \& McBride, B. W. Fatty acid composition of ruminal bacteria and protozoa, with emphasis on conjugated linoleic acid, vaccenic acid, and oddchain and branched-chain fatty acids1. J. Anim. Sci. 85, 1228-1234 (2007).

52. Dean, F. B. et al. Comprehensive human genome amplification using multiple displacement amplification. Proc. Natl. Acad. Sci. U. S. A. 99, 5261-5266 (2002).

53. Macaulay, I. C. et al. Separation and parallel sequencing of the genomes and transcriptomes of single cells using G\&amp;T-seq. Nat. Protoc. 11, 2081-2103 (2016).

54. Dehority, B. A. Ciliate protozoa. in Methods in Gut Microbial Ecology for Ruminants (eds. Makkar, H. P. S. \& McSweeney, C. S.) 67-78 (Springer-Verlag, 2005). doi:10.1007/1-4020-3791-0_6. 
55. Li, D. et al. MEGAHIT v1.0: A fast and scalable metagenome assembler driven by advanced methodologies and community practices. Methods 102, 3-11 (2016).

56. Bankevich, A. et al. SPAdes: a new genome assembly algorithm and its applications to single-cell sequencing. J. Comput. Biol. J. Comput. Mol. Cell Biol. 19, 455-477 (2012).

57. Chakraborty, M., Baldwin-Brown, J. G., Long, A. D. \& Emerson, J. J. Contiguous and accurate de novo assembly of metazoan genomes with modest long read coverage. Nucleic Acids Res. gkw654 (2016) doi:10.1093/nar/gkw654.

58. Bailey, T. L. et al. MEME Suite: tools for motif discovery and searching. Nucleic Acids Res. 37, W202-W208 (2009).

59. Parks, D. H. et al. Recovery of nearly 8,000 metagenome-assembled genomes substantially expands the tree of life. Nat. Microbiol. 2, 1533-1542 (2017).

60. Gilbert, R. A. et al. Toward Understanding Phage:Host Interactions in the Rumen; Complete Genome Sequences of Lytic Phages Infecting Rumen Bacteria. Front. Microbiol. 8, (2017).

61. Wang, L. et al. The transcriptome of the rumen ciliate Entodinium caudatum reveals some of its metabolic features. BMC Genomics 20, 1008 (2019).

62. Stanke, M. \& Morgenstern, B. AUGUSTUS: a web server for gene prediction in eukaryotes that allows user-defined constraints. Nucleic Acids Res. 33, W465-W467 (2005).

63. Haas, B. J. et al. De novo transcript sequence reconstruction from RNA-seq using the Trinity platform for reference generation and analysis. Nat. Protoc. 8, 1494-1512 (2013).

64. Pritchard, L., Glover, R. H., Humphris, S., Elphinstone, J. G. \& Toth, I. K. Genomics and taxonomy in diagnostics for food security: soft-rotting enterobacterial plant pathogens. Anal. Methods 8, 12-24 (2015).

65. Stamatakis, A. RAxML version 8: a tool for phylogenetic analysis and post-analysis of 
large phylogenies. Bioinformatics 30, 1312-1313 (2014).

789

790

66. Yang, Z. PAML 4: phylogenetic analysis by maximum likelihood. Mol. Biol. Evol. 24, 1586-1591 (2007).

67. Mendes, F. K., Vanderpool, D., Fulton, B. \& Hahn, M. W. CAFE 5 models variation in evolutionary rates among gene families. Bioinformatics 36, 5516-5518 (2020).

68. Huerta-Cepas, J. et al. eggNOG 5.0: a hierarchical, functionally and phylogenetically annotated orthology resource based on 5090 organisms and 2502 viruses. Nucleic Acids Res. 47, D309-D314 (2019).

69. Zhang, H. et al. dbCAN2: a meta server for automated carbohydrate-active enzyme annotation. Nucleic Acids Res. 46, W95-W101 (2018).

70. Minh, B. Q. et al. IQ-TREE 2: New Models and Efficient Methods for Phylogenetic Inference in the Genomic Era. Mol. Biol. Evol. 37, 1530-1534 (2020).

71. You, S. et al. Insight into the functional roles of Glu175 in the hyperthermostable xylanase XYL10C- $\Delta \mathrm{N}$ through structural analysis and site-saturation mutagenesis. Biotechnol. Biofuels 11, 159 (2018).

72. Bailey, M. J., Biely, P. \& Poutanen, K. Interlaboratory testing of methods for assay of xylanase activity. J. Biotechnol. 23, 257-270 (1992).

73. Yang, H. et al. Impact of disulfide bonds on the folding and refolding capability of a novel thermostable GH45 cellulase. Appl. Microbiol. Biotechnol. 102, 9183-9192 (2018).

74. He, H. et al. A Combinational Strategy for Effective Heterologous Production of Functional Human Lysozyme in Pichia pastoris. Front. Bioeng. Biotechnol. 8, 118 (2020).

75. Wood, D. E., Lu, J. \& Langmead, B. Improved metagenomic analysis with Kraken 2. Genome Biol. 20, 257 (2019).

76. Shen, J. et al. Metagenomic Analyses of Microbial and Carbohydrate-Active Enzymes in the Rumen of Dairy Goats Fed Different Rumen Degradable Starch. Front. Microbiol. 11, 
$8131003(2020)$.

814 77. Li, J. et al. A catalog of microbial genes from the bovine rumen unveils a specialized and

815 diverse biomass-degrading environment. GigaScience 9, (2020).

816 78. Dehority, B. A. Laboratory manual for classification and morphology of rumen ciliate

817 protozoa. (CRC Press, 1993).

818 
bioRxiv preprint doi: https://doi.org/10.1101/2022.01.05.474053; this version posted January 5, 2022. The copyright holder for this preprint (which was not certified by peer review) is the author/funder. All rights reserved. No reuse allowed without permission.

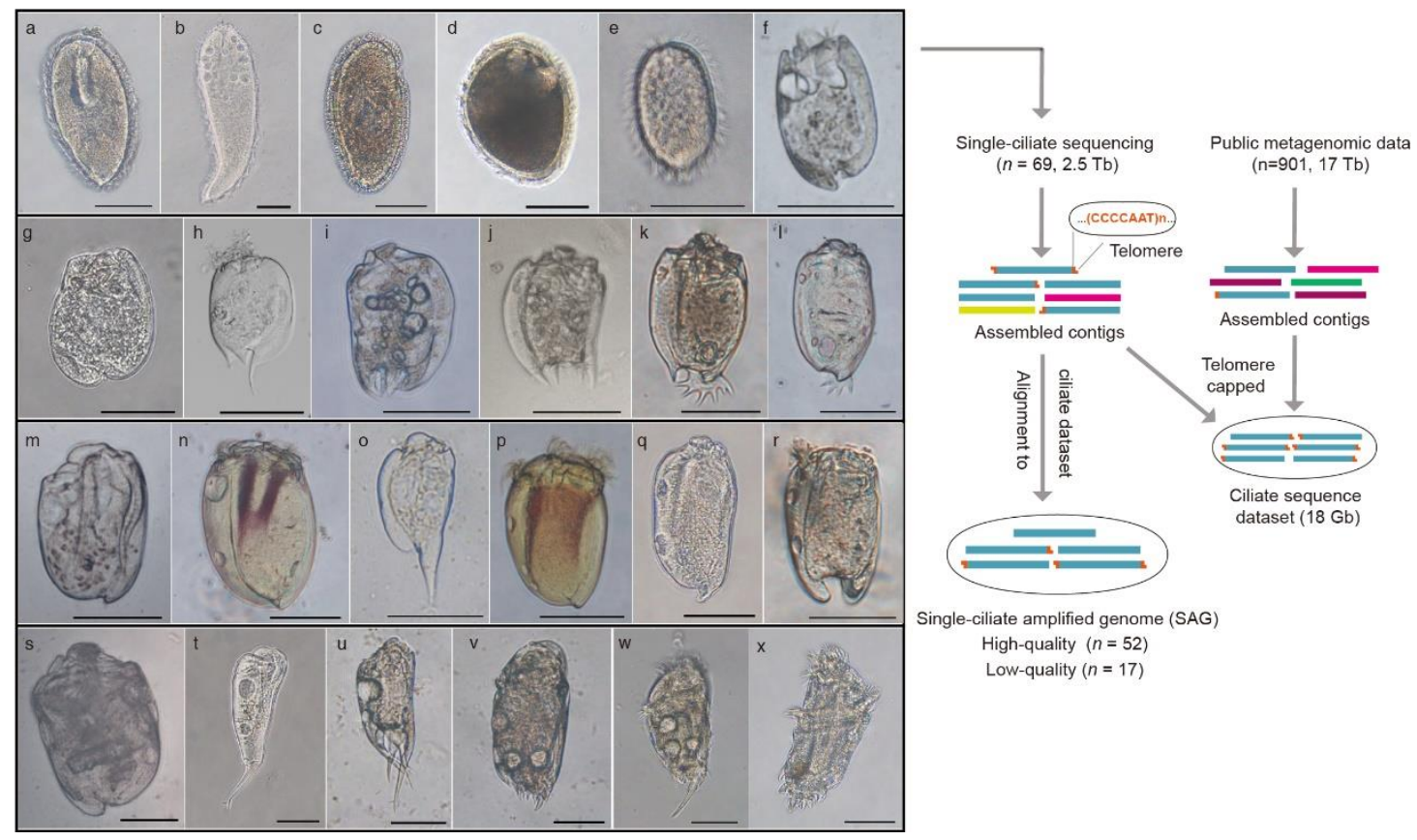

Fig. 1 

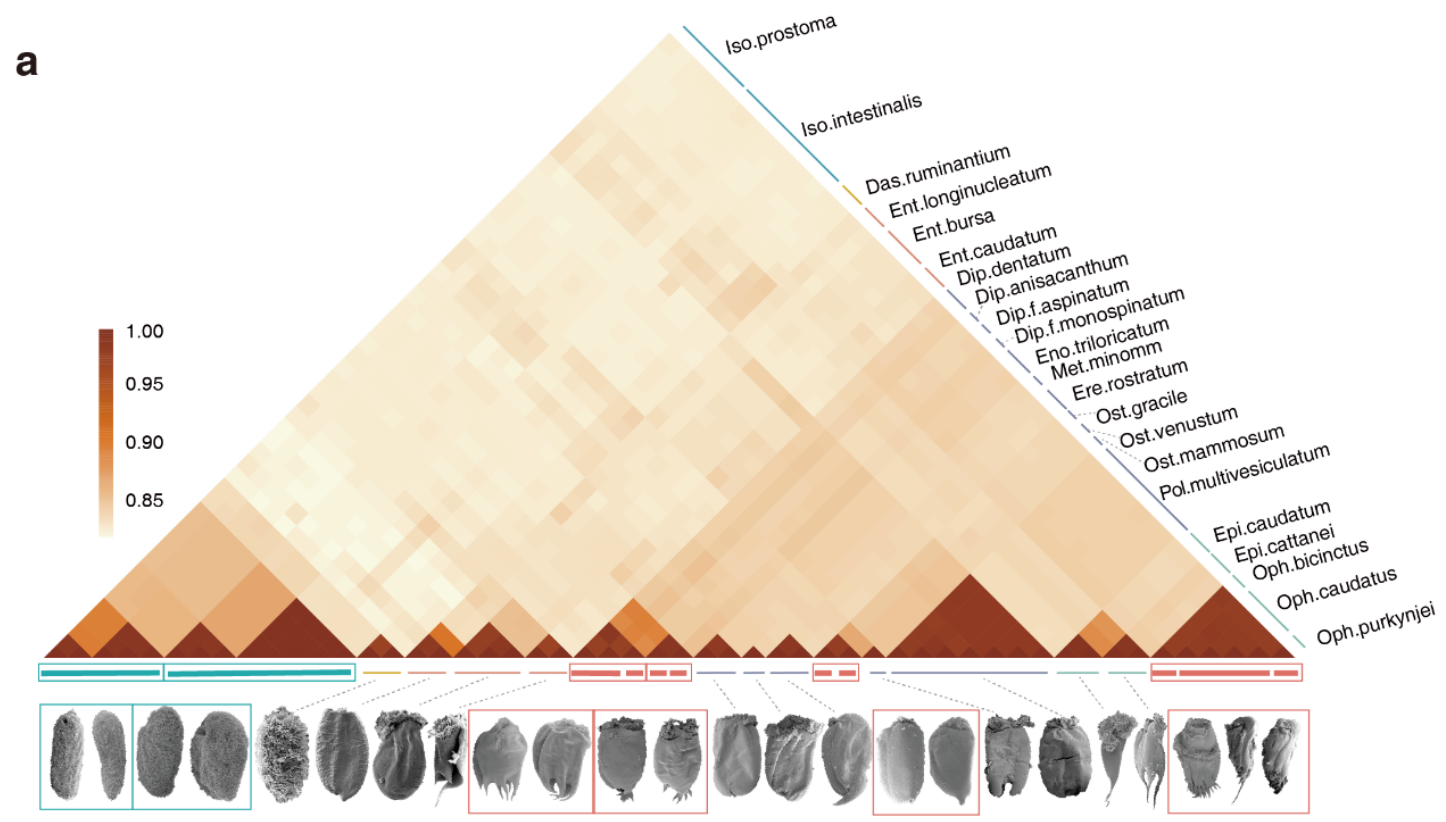

b
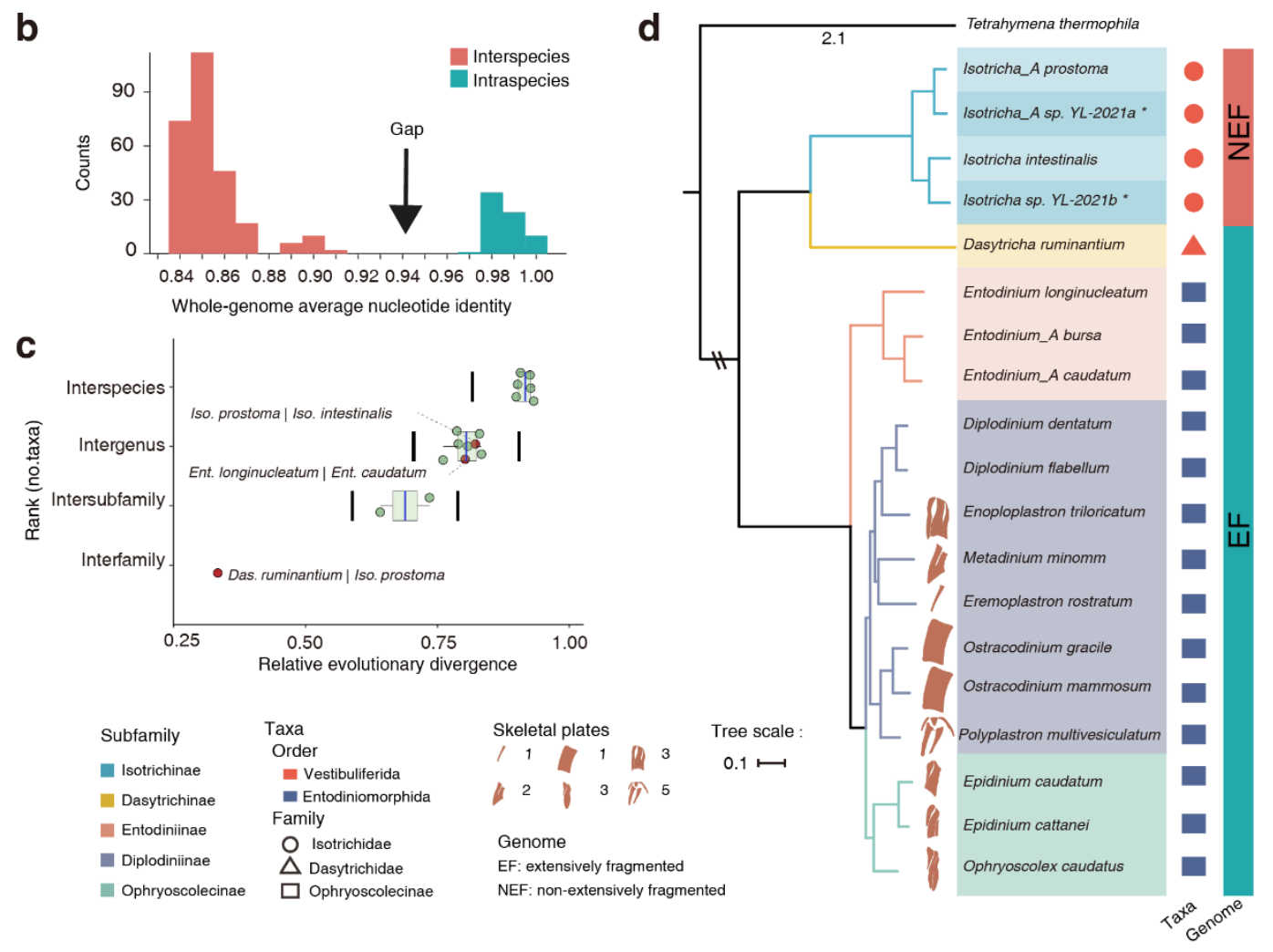

Fig. 2 
a
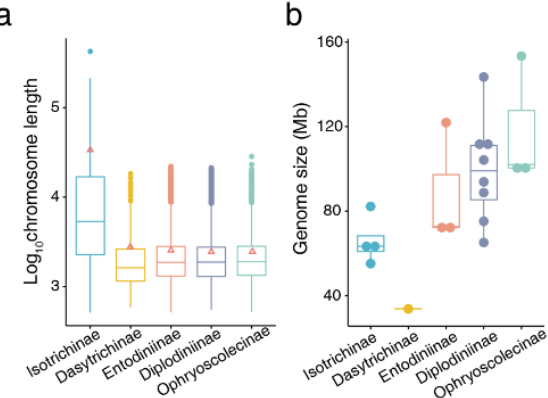

e

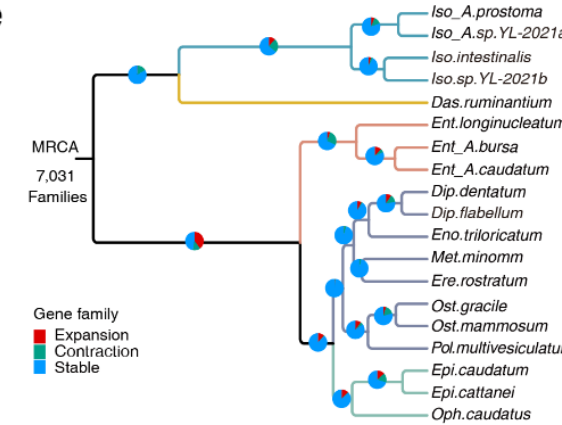

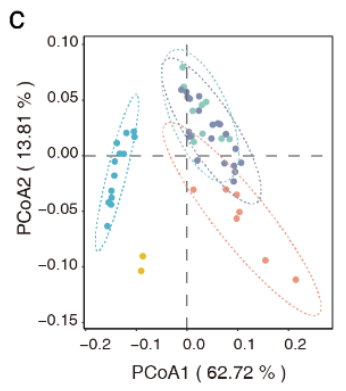
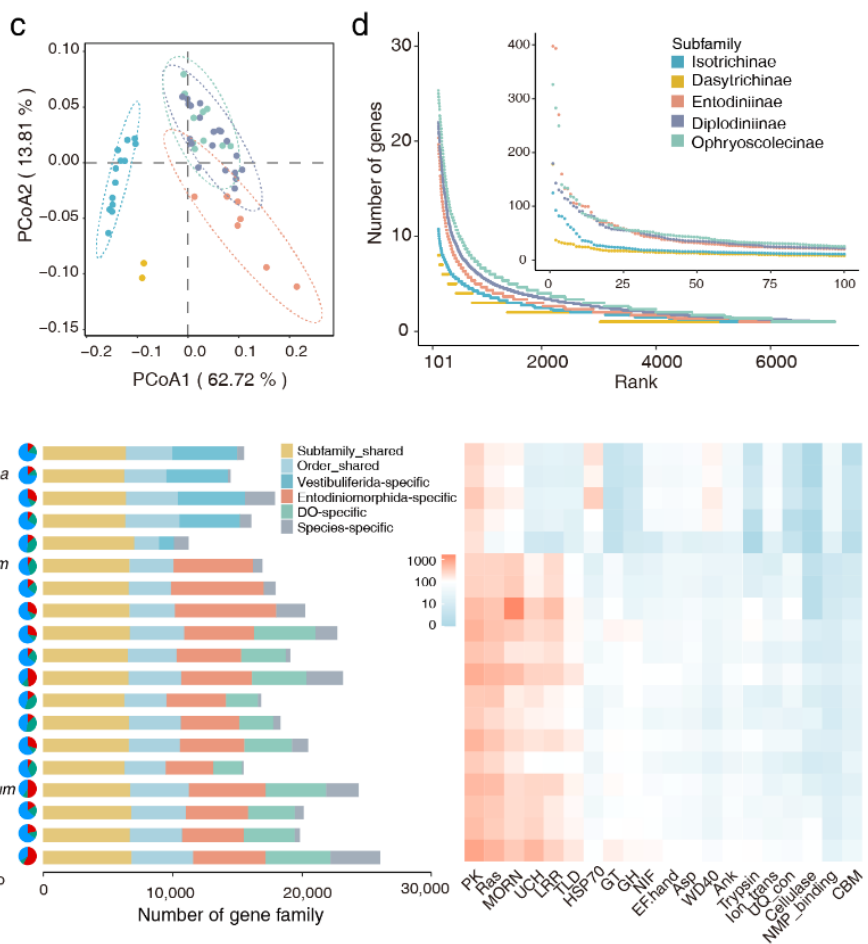

Fig. 3 
bioRxiv preprint doi: https://doi.org/10.1101/2022.01.05.474053; this version posted January 5, 2022. The copyright holder for this preprint (which was not certified by peer review) is the author/funder. All rights reserved. No reuse allowed without permission.

a

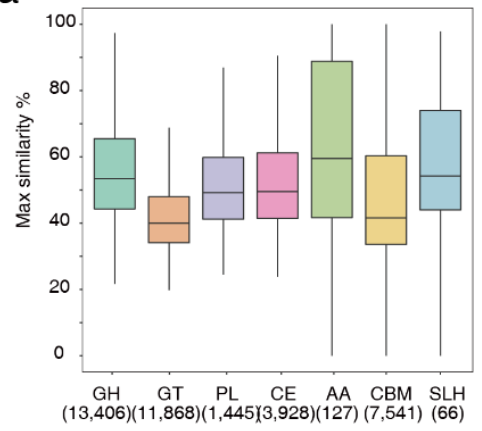

b

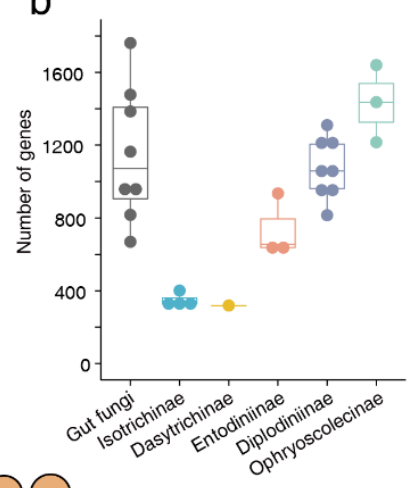

e

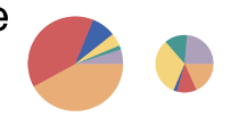

C

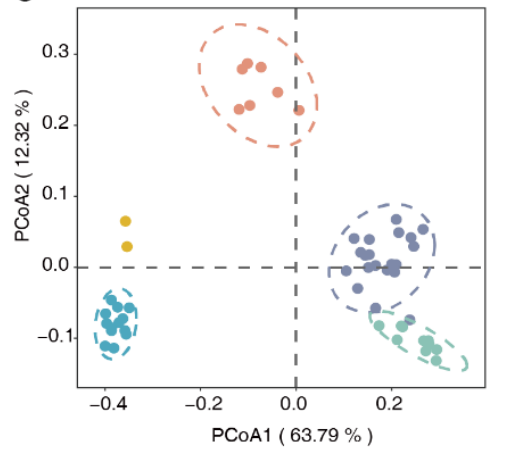

d

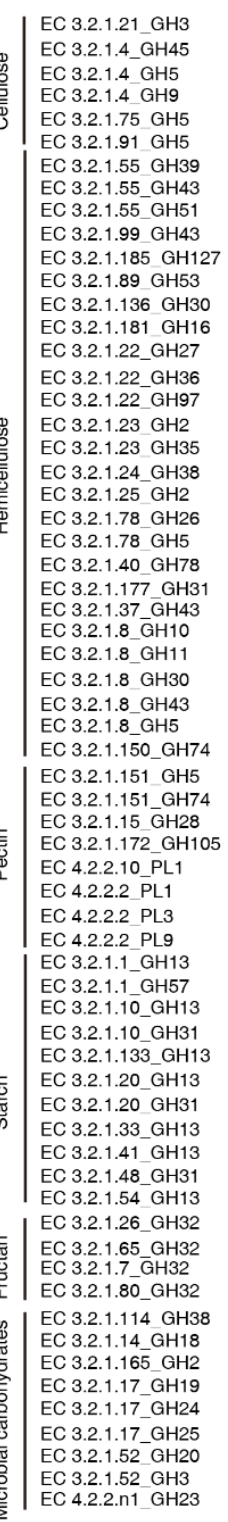

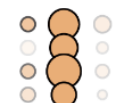

008
0
0

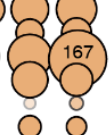

8 ०

000

- 00
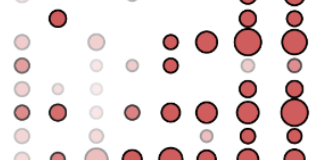

(

8

$\bigcirc$

00

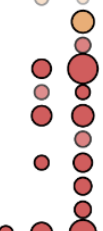

88

. 88

○

88

- 0 ○ 8

$\begin{array}{ll}\circ \\ \circ & \circ \\ \circ & 0 \\ 0 & 8\end{array}$

○

88

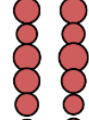

88
88
$\circ 8$
$\circ$

$\circ$

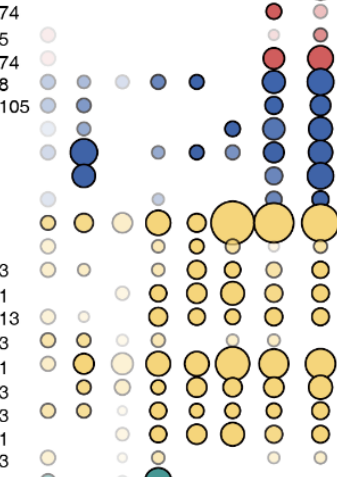

0

$\circ \circ \circ$

$\circ \circ 8$

0888

○ 80888

$\begin{array}{llll}\circ & \circ \\ \circ & 0 & 0 & 0 \\ 0 & 0 & 0 & 0\end{array}$
Substrate

Hemicellulose

O Pectin

Starch

Fructan

Microbial
carbohydrates

Gene number

(2) $4_{10}^{40}$

Prevalence

$25 \%$

$50 \%$

$75 \%$

g

$\frac{n=241}{\text { Gut fungi }} \frac{n=89}{\text { Isotrichinae Dasytrichinae }} \frac{n=182}{\text { Entodininae }} \frac{n=341}{\text { Diplodininae }} \quad \frac{n=522}{\text { Ophryoscolecinae }}$

f

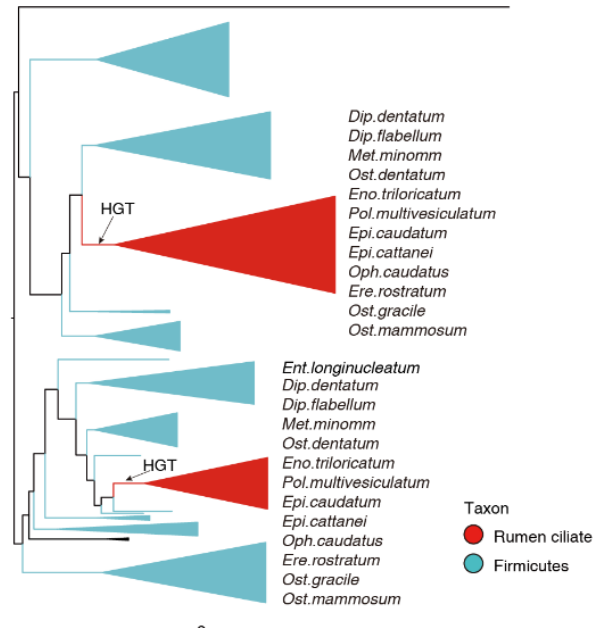

Motif locations of cellulase (EC 3.2.1.4_GH5)

9

(1)

Oph. caudatus $\square \square \square \square \square \square$

Motif locations of xylanase (EC 3.2.1.8_GH10)

Ost.gracile

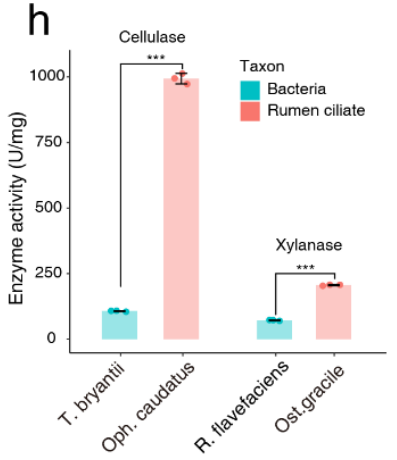

i

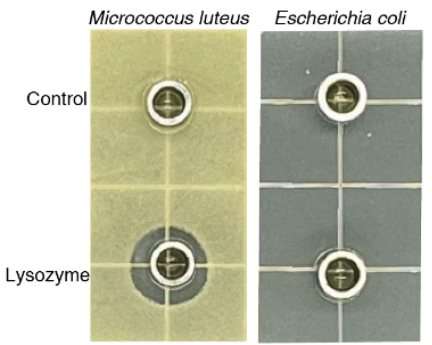

Fig. 4 

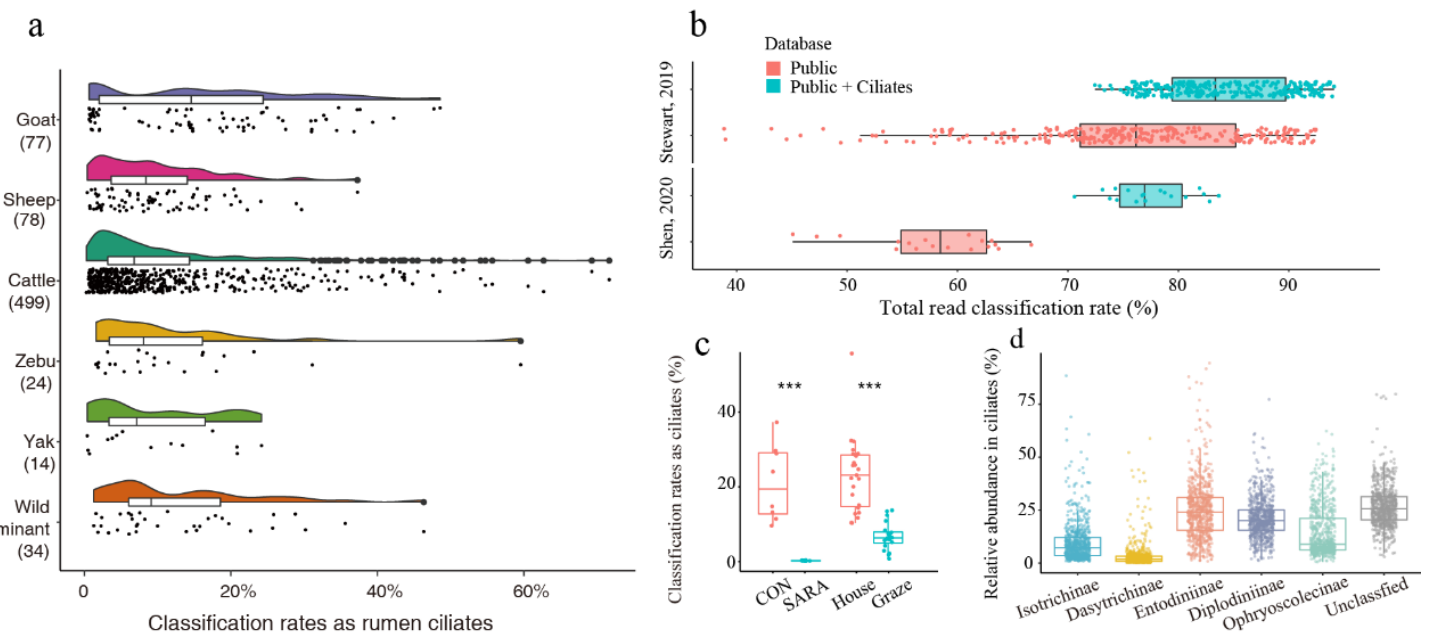

Fig. 5 